\title{
Computation of Topological Indices of Graphene
}

\author{
G. Sridhara, ${ }^{1,2}$ M. R. Rajesh Kanna, ${ }^{1}$ and R. S. Indumathi ${ }^{3}$ \\ ${ }^{1}$ Post Graduate Department of Mathematics, Maharani's Science College for Women, J. L. B. Road, Mysore 570005, India \\ ${ }^{2}$ Research and Development Centre, Bharathiar University, Coimbatore 641046, India \\ ${ }^{3}$ Maharaja Institute of Technology, Belawadi, Srirangapatna Taluk, Mandya, Karnataka 571438, India
}

Correspondence should be addressed to M. R. Rajesh Kanna; mr.rajeshkanna@gmail.com

Received 21 May 2015; Accepted 15 July 2015

Academic Editor: Stefano Bellucci

Copyright (C) 2015 G. Sridhara et al. This is an open access article distributed under the Creative Commons Attribution License, which permits unrestricted use, distribution, and reproduction in any medium, provided the original work is properly cited.

We compute $\mathrm{ABC}$ index, $\mathrm{ABC}_{4}$ index, Randic connectivity index, Sum connectivity index, GA index, and GA $\mathrm{A}_{5}$ index of Graphene.

\section{Introduction}

Graphene is an atomic scale honeycomb lattice made of carbon atoms. It is the world's first $2 \mathrm{D}$ material which was isolated from graphite in the year 2004 by Professor Andre Geim and Professor Kostya Novoselov. Graphene is 200 times stronger than steel, one million times thinner than a human hair, and world's most conductive material. So it has captured the attention of scientists, researchers, and industries worldwide. It is one of the most promising nanomaterials because of its unique combination of superb properties, which opens a way for its exploitation in a wide spectrum of applications ranging from electronics to optics, sensors, and biodevices. Also it is the most effective material for electromagnetic interference (EMI) shielding.

Topological indices are the molecular descriptors that describe the structures of chemical compounds and they help us to predict certain physicochemical properties like boiling point, enthalpy of vaporization, stability, and so forth. In this paper, we determine the topological indices like atom-bond connectivity index, fourth atom-bond connectivity index, Sum connectivity index, Randic connectivity index, geometric-arithmetic connectivity index, and fifth geometric-arithmetic connectivity index of Graphene.

All molecular graphs considered in this paper are finite, connected, loopless, and without multiple edges. Let $G=$ $(V, E)$ be a graph with $n$ vertices and $m$ edges. The degree of a vertex $u \in V(G)$ is denoted by $d_{u}$ and is the number of vertices that are adjacent to $u$. The edge connecting the vertices $u$ and $v$ is denoted by $u v$. Using these terminologies, certain topological indices are defined in the following manner.

The atom-bond connectivity index, $\mathrm{ABC}$ index, is one of the degree based molecular descriptors, which was introduced by Estrada et al. [1] in late 1990s, and it can be used for modelling thermodynamic properties of organic chemical compounds; it is also used as a tool for explaining the stability of branched alkanes [2]. Some upper bounds for the atombond connectivity index of graphs can be found in [3]. The atom-bond connectivity index of chemical bicyclic graphs and connected graphs can be seen in $[4,5]$. For further results on $\mathrm{ABC}$ index of trees, see the papers [6-9] and the references cited therein.

Definition 1. Let $G=(V, E)$ be a molecular graph, and $d_{u}$ is the degree of the vertex $u$; then $\mathrm{ABC}$ index of $G$ is defined as $\operatorname{ABC}(G)=\sum_{u v \in E} \sqrt{\left(d_{u}+d_{v}-2\right) / d_{u} d_{v}}$.

The fourth atom-bond connectivity index, $\mathrm{ABC}_{4}(G)$ index, was introduced by Ghorbani and Hosseinzadeh [10] in 2010. Further studies on $\mathrm{ABC}_{4}(G)$ index can be found in $[11,12]$.

Definition 2. Let $G$ be a graph; then its fourth $\mathrm{ABC}$ index is defined as $\mathrm{ABC}_{4}(G)=\sum_{u v \in E(G)} \sqrt{\left(S_{u}+S_{v}-2\right) / S_{u} S_{v}}$, where $S_{u}$ is sum of the degrees of all neighbours of vertex $u$ in $G$. In other words, $S_{u}=\sum_{u v \in E(G)} d_{v}$, similarly for $S_{v}$.

The first and oldest degree based topological index is Randic index [13] denoted by $\chi(G)$ and it was introduced by 
Milan Randic in 1975. It provides a quantitative assessment of branching of molecules.

Definition 3. For the graph $G$ Randic index is defined as $\chi(G)=\sum_{u v \in E(G)}\left(1 / \sqrt{d_{u} d_{v}}\right)$.

Sum connectivity index belongs to a family of Randic like indices and it was introduced by Zhou and Trinajstić [14]. Further studies on Sum connectivity index can be found in [15].

Definition 4. For a simple connected graph $G$, its Sum connectivity index $S(G)$ is defined as $S(G)=\sum_{u v \in E(G)}(1 /$ $\left.\sqrt{d_{u}+d_{v}}\right)$.

The geometric-arithmetic index, $\mathrm{GA}(G)$ index, of a graph $G$ was introduced by Vukičević and Furtula [16]. Further studies on GA index can be found in [17-19].

Definition 5. Let $G$ be a graph and let $e=u v$ be an edge of $G$; then, $\operatorname{GA}(G)=\sum_{e=u v \in E(G)}\left(2 \sqrt{d_{u} d_{v}} / d_{u}+d_{v}\right)$.

The fifth geometric-arithmetic index, $\mathrm{GA}_{5}(G)$, was introduced by Graovac et al. [20] in 2011.

Definition 6. For a graph $G$, the fifth geometric-arithmetic index is defined as $\mathrm{GA}_{5}(G)=\sum_{u v \in E(G)}\left(2 \sqrt{S_{u} S_{v}} /\left(S_{u}+S_{v}\right)\right)$, where $S_{u}$ is the sum of the degrees of all neighbors of the vertex $u$ in $G$, similarly $S_{v}$.

\section{Main Results}

Theorem 7. The atom-bond connectivity index of Graphene with " $t$ " rows of benzene rings and " $s$ " benzene rings in each row is given by

$A B C(G)$

$$
=\left\{\begin{array}{cc}
\frac{(12+2 \sqrt{2}) s+(6-2 \sqrt{2})}{3 \sqrt{2}}, & \text { if } t=1, \\
\frac{(12-4 \sqrt{2}) s+(9-2 \sqrt{2}) t+6 \sqrt{2} t s-2 \sqrt{2}}{3 \sqrt{2}}, & \text { if } t \neq 1 .
\end{array}\right.
$$

Proof. Consider a Graphene with " $t$ " rows and " $s$ " benzene rings in each row. Let $m_{i, j}$ denote the number of edges connecting the vertices of degrees $d_{i}$ and $d_{j}$. Two-dimensional structure of Graphene (as shown in Figure 1) contains only $m_{2,2}, m_{2,3}$, and $m_{3,3}$ edges. In Figure $1 m_{2,2}$ and $m_{3,3}$ edges are colored in green and red, respectively. The number of $m_{2,2}$, $m_{2,3}$, and $m_{3,3}$ edges in each row is mentioned in Table 1.

$\therefore$ Graphene contains $m_{2,2}=(t+4)$ edges, $m_{2,3}=(4 s+$ $2 t-4)$ edges, and $m_{3,3}=(3 t s-2 s-t-1)$ edges.

Case 1. The atom-bond connectivity index of Graphene for $t \neq 1$ is
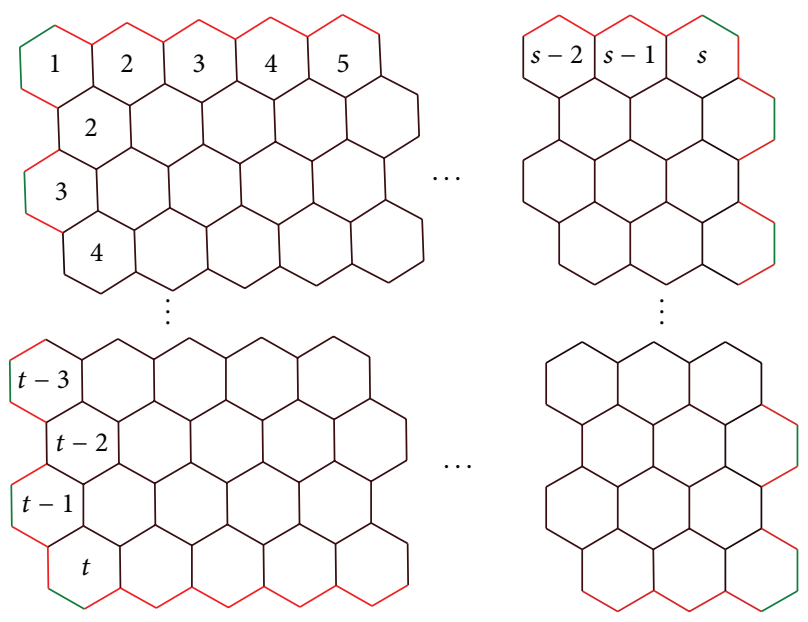

Figure 1

TABLE 1

\begin{tabular}{lccc}
\hline Row & $m_{2,2}$ & $m_{2,3}$ & $m_{3,3}$ \\
\hline 1 & 3 & $2 s$ & $3 s-2$ \\
2 & 1 & 2 & $3 s-1$ \\
3 & 1 & 2 & $3 s-1$ \\
4 & 1 & 2 & $3 s-1$ \\
$\vdots$ & $\vdots$ & $\vdots$ & $\vdots$ \\
$t$ & 3 & $2 s$ & $s-1$ \\
\hline Total & $t+4$ & $4 s+2 t-4$ & $3 t s-2 s-t-1$ \\
\hline
\end{tabular}

$$
\begin{aligned}
\mathrm{ABC} & (G)=\sum_{u v \in E} \sqrt{\frac{d_{u}+d_{v}-2}{d_{u} d_{v}}} \\
= & m_{2,2} \sqrt{\frac{2+2-2}{2.2}}+m_{2,3} \sqrt{\frac{2+3-2}{2.3}} \\
& +m_{3,3} \sqrt{\frac{3+3-2}{3.3}} \\
= & (t+4)\left(\frac{1}{\sqrt{2}}\right)+(4 s+2 t-4)\left(\frac{1}{\sqrt{2}}\right) \\
& +(3 t s-2 s-t-1)\left(\frac{2}{3}\right) \\
= & \left(\frac{1}{\sqrt{2}}\right)(t+4+4 s+2 t-4) \\
& +(6 t s-4 s-2 t-2)\left(\frac{1}{3}\right) \\
= & \left(\frac{1}{\sqrt{2}}\right)(3 t+4 s)+(6 t s-4 s-2 t-2)\left(\frac{1}{3}\right) \\
= & \frac{9 t+12 s+6 \sqrt{2} t s-4 \sqrt{2} s-2 \sqrt{2} t-2 \sqrt{2}}{3 \sqrt{2}}
\end{aligned}
$$




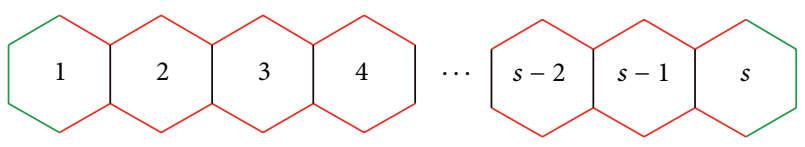

FIGURE 2

$\therefore \mathrm{ABC}(G)$

$$
=\frac{(12-4 \sqrt{2}) s+(9-2 \sqrt{2}) t+6 \sqrt{2} t s-2 \sqrt{2}}{3 \sqrt{2}},
$$

$t \neq 1$.

Case 2. For $t=1, m_{2,2}=6, m_{2,3}=(4 s-4)$, and $m_{3,3}=(s-1)$ edges as shown in Figure 2:

$$
\begin{aligned}
\operatorname{ABC}(G) & =\sum_{u v \in E} \sqrt{\frac{d_{u}+d_{v}-2}{d_{u} d_{v}}} \\
& =m_{2,2} \sqrt{\frac{2+2-2}{2.2}}+m_{2,3} \sqrt{\frac{2+3-2}{2.3}}
\end{aligned}
$$

$$
A B C_{4}(G)= \begin{cases}\frac{3 \sqrt{6}}{2}, & \text { if } t=1, s=1, \\ 2.541937 s+1.140265, & \text { if } t=1, s>1 \\ (1.217295) t+(1.212097) s+(1.333333) t s-(0.088070), & \text { if } t \neq 1 .\end{cases}
$$

Theorem 8. The fourth atom-bond connectivity index of Graphene is

$$
\begin{aligned}
& +m_{3,3} \sqrt{\frac{3+3-2}{3.3}} \\
= & 6\left(\frac{1}{\sqrt{2}}\right)+(4 s-4)\left(\frac{1}{\sqrt{2}}\right) \\
& +(s-1)\left(\frac{2}{3}\right) \\
= & (6+4 s-4)\left(\frac{1}{\sqrt{2}}\right)+(s-1)\left(\frac{2}{3}\right) \\
= & (4 s+2)\left(\frac{1}{\sqrt{2}}\right)+(s-1)\left(\frac{2}{3}\right) \\
= & \frac{12 s+6+2 \sqrt{2} s-2 \sqrt{2}}{3 \sqrt{2}}
\end{aligned}
$$$$
\therefore \operatorname{ABC}(G)=\frac{(12+2 \sqrt{2}) s+(6-2 \sqrt{2})}{3 \sqrt{2}}, \quad \text { for } t=1 \text {. }
$$

Proof. Let $e_{i, j}$ denote the number of edges of Graphene with $i=S_{u}$ and $j=S_{v}$. It is easy to see that the summation of degrees of edge endpoints of Graphene has nine edge types $e_{4,5}, e_{5,5}, e_{5,7}, e_{5,8}, e_{6,7}, e_{7,9}, e_{8,8}, e_{8,9}$, and $e_{9,9}$ that are enumerated in Table 2. For convenience these edge types are colored by grey, yellow, red, purple, blue, green, lightblue, brown, and black, respectively, as shown in Figure 3.

Case 1. The fourth atom-bond connectivity index of Graphene for $t \neq 1$ is

$$
\begin{aligned}
& \mathrm{ABC}_{4}(G)=\sum_{u v \in E(G)} \sqrt{\frac{S_{u}+S_{v}-2}{S_{u} S_{v}}}=e_{4,5}\left(\sqrt{\frac{7}{20}}\right) \\
& +e_{5,5}\left(\frac{\sqrt{8}}{5}\right)+e_{5,7}\left(\sqrt{\frac{2}{7}}\right)+e_{5,8}\left(\sqrt{\frac{11}{40}}\right) \\
& +e_{6,7}\left(\sqrt{\frac{11}{42}}\right)+e_{7,9}\left(\sqrt{\frac{14}{63}}\right)+e_{8,8}\left(\frac{\sqrt{14}}{8}\right) \\
& +e_{9,8}\left(\sqrt{\frac{15}{72}}\right)+e_{9,9}\left(\frac{4}{9}\right)=4\left(\sqrt{\frac{7}{20}}\right)+t\left(\frac{\sqrt{8}}{5}\right)
\end{aligned}
$$

$$
\begin{aligned}
& +8\left(\sqrt{\frac{2}{7}}\right)+(2 t-4)\left(\sqrt{\frac{11}{40}}\right)+(4 s-8)\left(\sqrt{\frac{11}{42}}\right) \\
& +2 s\left(\sqrt{\frac{14}{63}}\right)+(t-2)\left(\frac{\sqrt{14}}{8}\right)+(2 t-4)\left(\sqrt{\frac{15}{72}}\right) \\
& +(3 t s-4 s-4 t+5)\left(\frac{4}{9}\right)=4\left(\frac{\sqrt{7}}{2 \sqrt{5}}\right)+t\left(\frac{\sqrt{8}}{5}\right) \\
& +8\left(\sqrt{\frac{2}{7}}\right)+2(t-2)\left(\frac{\sqrt{11}}{2 \sqrt{10}}\right)+(4 s-8)\left(\sqrt{\frac{11}{42}}\right) \\
& +2 s\left(\frac{\sqrt{2}}{3}\right)+(t-2)\left(\frac{\sqrt{14}}{8}\right)+(2 t-4)\left(\frac{\sqrt{5}}{2 \sqrt{6}}\right) \\
& +(3 t s-4 s-4 t+5)\left(\frac{4}{9}\right)=\left(\frac{\sqrt{8}}{5}+\sqrt{\frac{11}{10}}+\frac{\sqrt{14}}{8}\right. \\
& \left.+\sqrt{\frac{5}{6}}-\frac{16}{9}\right) t+\left(4 \sqrt{\frac{11}{42}}+\frac{2 \sqrt{2}}{3}-\frac{16}{9}\right) s+\frac{4}{3} t s \\
& +\left(2 \sqrt{\frac{7}{5}}+8 \sqrt{\frac{2}{7}}-2 \sqrt{\frac{11}{10}}-8 \sqrt{\frac{11}{42}}-\frac{\sqrt{14}}{4}-2 \sqrt{\frac{5}{6}}\right.
\end{aligned}
$$


TABle 2

\begin{tabular}{lccccccccc}
\hline Rows & $e_{4,5}$ & $e_{5,5}$ & $e_{5,7}$ & $e_{5,8}$ & $e_{6,7}$ & $e_{7,9}$ & $e_{8,8}$ & $e_{8,9}$ & $e_{9,9}$ \\
\hline 1 & 2 & 1 & 3 & 1 & $2 s-4$ & $s$ & 0 & 1 & $2 s-3$ \\
2 & 0 & 1 & 1 & 1 & 0 & 0 & 1 & 2 & $3 s-4$ \\
3 & 0 & 1 & 0 & 2 & 0 & 0 & 1 & 2 & $3 s-4$ \\
4 & 0 & 1 & 0 & 2 & 0 & 0 & 1 & 2 & $3 s-4$ \\
$\vdots$ & $\vdots$ & $\vdots$ & $\vdots$ & $\vdots$ & $\vdots$ & $\vdots$ & $\vdots$ & $\vdots$ & $\vdots$ \\
$t-2$ & 0 & 1 & 0 & 2 & 0 & 0 & 1 & 2 & $3 s-4$ \\
$t-1$ & 0 & 1 & 1 & 1 & 0 & 0 & 1 & 1 & $3 s-4$ \\
$t$ & 2 & 1 & 3 & 1 & $2 s-4$ & $s$ & 0 & 0 & 0 \\
\hline \multirow{2}{*}{ Total } & 4 & $t$ & 8 & $2 t-4$ & $4 s-8$ & $2 s$ & $t-2$ & $2 t-4$ & $3 t s-4 s-$ \\
& & & & & & & & & \\
\end{tabular}

$\left.+\frac{20}{9}\right)$

$\mathrm{ABC}_{4}(G)=(1.217295) t+(1.212097) s+(1.333333)$

$\cdot t s-(0.088070), \quad t \neq 1$.

(5)

Case 2. For $t=1$ and $s>1$, Graphene has five types of edges, namely, $e_{4,4}, e_{4,5}, e_{5,7}, e_{6,7}$, and $e_{7,7}$. These edges are colored in orange, pink, red, blue, and lavender, respectively, as shown in Figure 4 . The number of edges of these types is shown in Table 3:

$$
\begin{aligned}
\mathrm{ABC}_{4}(G) \\
=e_{4,4}\left(\frac{\sqrt{6}}{4}\right)+e_{4,5}\left(\sqrt{\frac{7}{20}}\right)+e_{5,7}\left(\sqrt{\frac{2}{7}}\right) \\
+e_{6,7}\left(\sqrt{\frac{11}{42}}\right)+e_{7,7}\left(\frac{\sqrt{12}}{7}\right) \\
=2\left(\frac{\sqrt{6}}{4}\right)+4\left(\sqrt{\frac{7}{20}}\right)+4\left(\sqrt{\frac{2}{7}}\right) \\
+(4 s-8) \sqrt{\frac{11}{42}}+(s-1)\left(\frac{\sqrt{12}}{7}\right) \\
=\left(4 \sqrt{\frac{11}{42}}+\frac{\sqrt{12}}{7}\right) s \\
+\left(\frac{\sqrt{6}}{2}+4 \sqrt{\frac{7}{20}}+4 \sqrt{\frac{2}{7}}-8 \sqrt{\frac{11}{42}}-\frac{\sqrt{12}}{7}\right) \\
\therefore \mathrm{ABC}_{4}(G)=(2.541937) s+1.140265 .
\end{aligned}
$$

Case 3. For $t=1$ and $s=1$, we have only 6 edges of the type $e_{4,4}$ as shown in Figure 5:
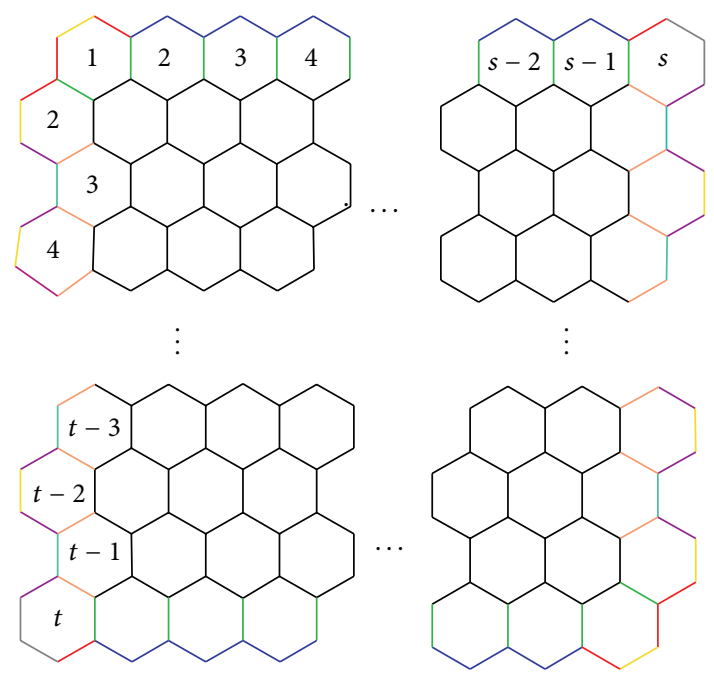

Figure 3

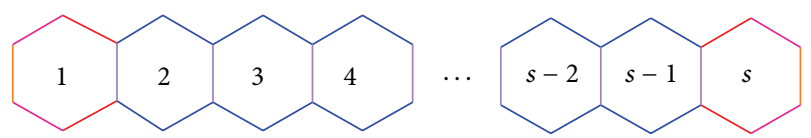

Figure 4

$$
\begin{aligned}
\mathrm{ABC}_{4}(G) & =e_{4,4}\left(\frac{\sqrt{6}}{4}\right)=\frac{6 \sqrt{6}}{4}, \\
\therefore \mathrm{ABC}_{4}(G) & =\frac{3 \sqrt{6}}{2} .
\end{aligned}
$$

Theorem 9. The Randic connectivity index of Graphene is

$$
\begin{aligned}
& \chi(G) \\
& =\left\{\begin{array}{cl}
\frac{(2 \sqrt{6}+1) s+(8-2 \sqrt{6})}{3}, & \text { if } t=1, \\
\frac{(1+2 \sqrt{6}) t-4(1-\sqrt{6}) s+6 t s+(10-4 \sqrt{6})}{6}, & \text { if } t \neq 1 .
\end{array}\right.
\end{aligned}
$$

Proof.

Case 1 . For $t \neq 1$,

$$
\begin{aligned}
& \chi(G)=\sum_{e=u v \in E(G)} \frac{1}{\sqrt{d_{u} d_{v}}} \\
& \quad=m_{2,2}\left(\frac{1}{\sqrt{2.2}}\right)+m_{2,3}\left(\frac{1}{\sqrt{3.2}}\right)+m_{3,3}\left(\frac{1}{\sqrt{3.3}}\right)
\end{aligned}
$$




$$
\begin{aligned}
& \checkmark \\
& \text { Case 2. For } t=1 \text {, } \\
& \text { Figure } 5 \\
& =(t+4)\left(\frac{1}{2}\right)+(4 s+2 t-4)\left(\frac{1}{\sqrt{6}}\right) \\
& +(3 t s-2 s-t-1)\left(\frac{1}{3}\right) \\
& =\left(\frac{3 t+12+6 t s-4 s-2 t-2}{6}\right) \\
& +(4 s+2 t-4)\left(\frac{1}{\sqrt{6}}\right) \\
& =\left(\frac{t-4 s+6 t s+10}{6}\right)+(4 s+2 t-4)\left(\frac{1}{\sqrt{6}}\right) \\
& =\frac{(t-4 s+6 t s+10)+\sqrt{6}(4 s+2 t-4)}{6} \\
& =\frac{t-4 s+6 t s+10+4 \sqrt{6} s+2 \sqrt{6} t-4 \sqrt{6}}{6} \\
& \therefore \chi(G) \\
& =\frac{(1+2 \sqrt{6}) t-4(1-\sqrt{6}) s+6 t s+(10-4 \sqrt{6})}{6} . \\
& \chi(G)=\sum_{e=u v \in E(G)} \frac{1}{\sqrt{d_{u} d_{v}}} \\
& =m_{2,2}\left(\frac{1}{\sqrt{2.2}}\right)+m_{2,3}\left(\frac{1}{\sqrt{3.2}}\right) \\
& +m_{3,3}\left(\frac{1}{\sqrt{3.3}}\right) \\
& =6\left(\frac{1}{2}\right)+(4 s-4)\left(\frac{1}{\sqrt{6}}\right)+(s-1)\left(\frac{1}{3}\right) \\
& =3+(4 s-4)\left(\frac{1}{\sqrt{6}}\right)+(s-1)\left(\frac{1}{3}\right) \\
& =\frac{9 \sqrt{6}+12 s-12+\sqrt{6} s-\sqrt{6}}{3 \sqrt{6}} \\
& \therefore \chi(G)=\frac{(2 \sqrt{6}+1) s+(8-2 \sqrt{6})}{3} .
\end{aligned}
$$

Theorem 10. The Sum connectivity index of Graphene is

$$
S(G)=\left\{\begin{array}{cc}
\frac{(\sqrt{5}+4 \sqrt{6}) s+(3 \sqrt{30}-\sqrt{5}-4 \sqrt{6})}{\sqrt{30}}, & \text { if } t=1, \\
\frac{(\sqrt{30}+4 \sqrt{6}-2 \sqrt{5}) t+(8 \sqrt{6}-4 \sqrt{5}) s+6 \sqrt{5} t s+(4 \sqrt{30}-8 \sqrt{6}-2 \sqrt{5})}{2 \sqrt{30}}, & \text { if } t \neq 1 .
\end{array}\right.
$$

Proof.

Case 1. For $t \neq 1$,

$$
\begin{aligned}
S(G) & =\sum_{e=u v \in E(G)} \frac{1}{\sqrt{d_{u}+d_{v}}}=m_{2,2}\left(\frac{1}{\sqrt{2+2}}\right)+m_{2,3}\left(\frac{1}{\sqrt{2+3}}\right)+m_{3,3}\left(\frac{1}{\sqrt{3+3}}\right) \\
& =(t+4)\left(\frac{1}{2}\right)+(4 s+2 t-4)\left(\frac{1}{\sqrt{5}}\right)+(3 t s-2 s-t-1)\left(\frac{1}{\sqrt{6}}\right) \\
& =\frac{t \sqrt{30}+4 \sqrt{30}+8 \sqrt{6} s+4 \sqrt{6} t-8 \sqrt{6}+6 \sqrt{5} t s-4 \sqrt{5} s-2 \sqrt{5} t-2 \sqrt{5}}{2 \sqrt{30}} \\
\therefore S(G) & =\frac{(\sqrt{30}+4 \sqrt{6}-2 \sqrt{5}) t+(8 \sqrt{6}-4 \sqrt{5}) s+6 \sqrt{5} t s+(4 \sqrt{30}-8 \sqrt{6}-2 \sqrt{5})}{2 \sqrt{30}}, \quad t \neq 1 .
\end{aligned}
$$


TABle 3

\begin{tabular}{lccccc}
\hline $\begin{array}{l}\text { Number of } \\
\text { benzene rings } \\
(s)\end{array}$ & $e_{4,4}$ & $e_{4,5}$ & $e_{5,7}$ & $e_{6,7}$ & $e_{7,7}$ \\
\hline 2 & 2 & 4 & 4 & 0 & 1 \\
3 & 2 & 4 & 4 & 4 & 2 \\
4 & 2 & 4 & 4 & 8 & 3 \\
5 & 2 & 4 & 4 & 12 & 4 \\
$\vdots$ & $\vdots$ & $\vdots$ & $\vdots$ & $\vdots$ & $\vdots$ \\
$s-1$ & 2 & 4 & 4 & $4 s-12$ & $s-2$ \\
$s$ & 2 & 4 & 4 & $4 s-8$ & $s-1$ \\
\hline
\end{tabular}

Case 2. For $t=1$,

$$
\begin{aligned}
S(G) & =\sum_{e=u v \in E(G)} \frac{1}{\sqrt{d_{u}+d_{v}}} \\
& =m_{2,2}\left(\frac{1}{2}\right)+m_{2,3}\left(\frac{1}{\sqrt{5}}\right)+m_{3,3}\left(\frac{1}{\sqrt{6}}\right) \\
& =6\left(\frac{1}{2}\right)+(4 s-4)\left(\frac{1}{\sqrt{5}}\right)+(s-1)\left(\frac{1}{\sqrt{6}}\right) \\
& =3+(4 s-4)\left(\frac{1}{\sqrt{5}}\right)+(s-1)\left(\frac{1}{\sqrt{6}}\right) \\
& =\left(\frac{3 \sqrt{6}+(s-1)}{\sqrt{6})+\left(\frac{4 s-4}{\sqrt{5}}\right)}\right. \\
& =\frac{3 \sqrt{30}+\sqrt{5} s-\sqrt{5}+4 \sqrt{6} s-4 \sqrt{6}}{\sqrt{30}} \\
\therefore S(G) & =\frac{(\sqrt{5}+4 \sqrt{6}) s+(3 \sqrt{30}-\sqrt{5}-4 \sqrt{6})}{\sqrt{30}} .
\end{aligned}
$$

Theorem 11. The geometric-arithmetic index of Graphene with " $t$ " rows and " $s$ " benzene rings in each row is given by

$G A(G)$

$$
=\left\{\begin{array}{cc}
\frac{(5+8 \sqrt{6}) s+(25-8 \sqrt{6})}{5}, & \text { if } t=1, \\
\frac{(8 \sqrt{6}-10) s+4 \sqrt{6} t+15 t s+(15-8 \sqrt{6})}{5}, & \text { if } t \neq 1 .
\end{array}\right.
$$

Proof.

Case 1. For $t \neq 1$,

$$
\begin{aligned}
\mathrm{GA} & (G)=\sum_{e=u v \in E(G)} \frac{2 \sqrt{d_{u} d_{v}}}{d_{u}+d_{v}} \\
& =m_{2,2}\left(\frac{2 \sqrt{2.2}}{2+2}\right)+m_{2,3}\left(\frac{2 \sqrt{2.3}}{2+3}\right)
\end{aligned}
$$

$$
\begin{aligned}
& +m_{3,3}\left(\frac{2 \sqrt{3.3}}{3+3}\right) \\
= & m_{2,2}+m_{2,3}\left(\frac{2 \sqrt{6}}{2+3}\right)+m_{3,3}\left(\frac{2(3)}{6}\right) \\
= & m_{2,2}+m_{2,3}\left(\frac{2 \sqrt{6}}{5}\right)+m_{3,3} \\
= & (t+4)+(4 s+2 t-4)\left(\frac{2 \sqrt{6}}{5}\right) \\
& +(3 t s-2 s-t-1) \\
= & \frac{(8 \sqrt{6}-10) s+4 \sqrt{6} t+15 t s+(15-8 \sqrt{6})}{5},
\end{aligned}
$$

$\mathrm{GA}(G)$

$$
=\frac{(8 \sqrt{6}-10) s+4 \sqrt{6} t+15 t s+(15-8 \sqrt{6})}{5}
$$

Case 2. For $t=1$,

$$
\begin{aligned}
\mathrm{GA}(G)= & \sum_{e=u v \in E(G)} \frac{2 \sqrt{d_{u} d_{v}}}{d_{u} d_{v}} \\
= & m_{2,2}\left(\frac{2 \sqrt{2.2}}{2+2}\right)+m_{2,3}\left(\frac{2 \sqrt{2.3}}{2+3}\right) \\
& +m_{3,3}\left(\frac{2 \sqrt{3.3}}{3+3}\right) \\
= & 6(1)+(4 s-4)\left(\frac{2 \sqrt{6}}{5}\right)+(s-1)(1) \\
= & (s+5)+(8 s-8)\left(\frac{\sqrt{6}}{5}\right) \\
= & \frac{5 s+25+8 \sqrt{6} s-8 \sqrt{6}}{5} \\
\operatorname{GA}(G)= & \frac{(5+8 \sqrt{6}) s+(25-8 \sqrt{6})}{5}, \quad \text { for } t=1 .
\end{aligned}
$$

Theorem 12. The fifth geometric-arithmetic index of Graphene is

$$
\begin{aligned}
& G A_{5}(G) \\
& = \begin{cases}6, & \text { if } t=1, s=1, \\
(4.988148) s+0.942989, & \text { if } t=1, s>1, \\
(1.942554) t+(1.972462) s+3 t s-0.998066, & \text { if } t \neq 1 .\end{cases}
\end{aligned}
$$


Proof.

Case 1. For $t \neq 1$,

$$
\begin{aligned}
& \mathrm{GA}_{5}(G)=\sum_{u v \in E(G)} \frac{2 \sqrt{S_{u} S_{v}}}{S_{u}+S_{v}}=e_{4,5}\left(\frac{4 \sqrt{5}}{9}\right)+e_{5,5}(1) \\
& +e_{5,7}\left(\frac{\sqrt{35}}{6}\right)+e_{5,8}\left(\frac{2 \sqrt{40}}{13}\right)+e_{6,7}\left(\frac{2 \sqrt{42}}{13}\right) \\
& +e_{7,9}\left(\frac{3 \sqrt{7}}{8}\right)+e_{8,8}(1)+e_{9,8}\left(\frac{12 \sqrt{2}}{17}\right)+e_{9,9}(1) \\
& =4\left(\frac{4 \sqrt{5}}{9}\right)+t(1)+8\left(\frac{\sqrt{35}}{6}\right)+(2 t-4) \\
& \cdot\left(\frac{2 \sqrt{40}}{13}\right)+(4 s-8)\left(\frac{2 \sqrt{42}}{13}\right)+2 s\left(\frac{3 \sqrt{7}}{8}\right)+(t \\
& -2)(1)+(2 t-4)\left(\frac{12 \sqrt{2}}{17}\right)+(3 t s-4 s-4 t+5) \\
& \cdot(1)=16\left(\frac{\sqrt{5}}{9}\right)+t+4\left(\frac{\sqrt{35}}{3}\right)+(4 t-8) \\
& \cdot\left(\frac{\sqrt{40}}{13}\right)+(8 s-16)\left(\frac{\sqrt{42}}{13}\right)+\left(\frac{3 \sqrt{7}}{4}\right) s+(t \\
& -2)+(24 t-48)\left(\frac{\sqrt{2}}{17}\right)+(3 t s-4 s-4 t+5) \\
& =\left[1+4\left(\frac{\sqrt{40}}{13}\right)+1+24\left(\frac{\sqrt{2}}{17}\right)-4\right] t \\
& +\left[8\left(\frac{\sqrt{42}}{13}\right)+3\left(\frac{\sqrt{7}}{4}\right)-4\right] s+3 t s \\
& +\left[16\left(\frac{\sqrt{5}}{9}\right)+4\left(\frac{\sqrt{35}}{3}\right)-8\left(\frac{\sqrt{40}}{13}\right)\right. \\
& \left.-16\left(\frac{\sqrt{42}}{13}\right)-2-48\left(\frac{\sqrt{2}}{17}\right)+5\right]=\left[\frac{4 \sqrt{40}}{13}\right. \\
& \left.+\frac{24 \sqrt{2}}{17}-2\right] t+\left[\frac{8 \sqrt{42}}{13}+\frac{3 \sqrt{7}}{4}-4\right] s+3 t s \\
& +\left[\frac{16 \sqrt{5}}{9}+\frac{4 \sqrt{35}}{3}-\frac{8 \sqrt{40}}{13}-\frac{16 \sqrt{42}}{13}-\frac{48 \sqrt{2}}{17}\right. \\
& +3] \text {. } \\
& \therefore \mathrm{GA}_{5}(G)=(1.942554) t+(1.972462) s+3 t s \\
& -0.998066, \quad t \neq 1 \text {. }
\end{aligned}
$$

Case 3. For $t=1$ and $s=1$, we have only 6 edges of the type $e_{4,4}$ as shown in Figure 5:

$$
\begin{aligned}
\mathrm{GA}_{5}(G) & =e_{4,4}(1)=6(1) \\
\therefore \mathrm{GA}_{5}(G) & =6 .
\end{aligned}
$$

\section{Conclusion}

The problem of finding the general formula for $\mathrm{ABC}$ index, $\mathrm{ABC}_{4}$ index, Randic connectivity index, Sum connectivity index, GA index, and $\mathrm{GA}_{5}$ index of Graphene is solved here analytically without using computers.

\section{Conflict of Interests}

The authors declare that there is no conflict of interests regarding the publication of this paper.

\section{Acknowledgments}

The authors are thankful to both anonymous referees for their valuable comments and useful suggestions. The second author is also thankful to the University Grants Commission, Government of India, for the financial support under the Grant MRP(S)-0535/13-14/KAMY004/UGC-SWRO. 


\section{References}

[1] E. Estrada, L. Torres, L. Rodríguez, and I. Gutman, "An atombond connectivity index: modelling the enthalpy of formation of alkanes," Indian Journal of Chemistry-Section A: Inorganic, Physical, Theoretical and Analytical Chemistry, vol. 37, no. 10, pp. 849-855, 1998.

[2] E. Estrada, "Atom-bond connectivity and the energetic of branched alkanes," Chemical Physics Letters, vol. 463, no. 4-6, pp. 422-425, 2008.

[3] J. Chen, J. Liu, and X. Guo, "Some upper bounds for the atom-bond connectivity index of graphs," Applied Mathematics Letters, vol. 25, no. 7, pp. 1077-1081, 2012.

[4] J.-S. Chen and X.-F. Guo, "The atom-bond connectivity index of chemical bicyclic graphs," Applied Mathematics. A Journal of Chinese Universities. Series B, vol. 27, no. 2, pp. 243-252, 2012.

[5] R. Xing, B. Zhou, and F. Dong, "On atom-bond connectivity index of connected graphs," Discrete Applied Mathematics, vol. 159, no. 15, pp. 1617-1630, 2011.

[6] B. Furtula, A. Graovac, and D. Vukicevic, "Atom-bond connectivity index of trees," Discrete Applied Mathematics., vol. 157, no. 13, pp. 2828-2835, 2009.

[7] I. Gutman, B. Furtula, and M. Ivanovic, "Notes on trees with minimal atom-bond connectivity index," MATCH Communications in Mathematical and in Computer Chemistry, vol. 67, no. 2, pp. 467-482, 2012.

[8] R. Xing, B. Zhou, and Z. Du, "Further results on atom-bond connectivity index of trees," Discrete Applied Mathematics, vol. 158, no. 14, pp. 1536-1545, 2010.

[9] R. Xing and B. Zhou, "Extremal trees with fixed degree sequence for atom-bond connectivity index," Filomat, vol. 26, no. 4, pp. 683-688, 2012.

[10] M. Ghorbani and M. A. Hosseinzadeh, "Computing ABC4 index of nanostar dendrimers," Optoelectronics and Advanced Materials, Rapid Communications, vol. 4, no. 9, pp. 1419-1422, 2010.

[11] M. R. Farahani, "Computing fourth atom-bond connectivity index of V-Phenylenic Nanotubes and Nanotori," Acta Chimica Slovenica, vol. 60, no. 2, pp. 429-432, 2013.

[12] M. R. Farahani, "On the fourth atom-bond connectivity index of armchair polyhex nanotubes," Proceedings of the Romanian Academy, Series B, vol. 15, no. 1, pp. 3-6, 2013.

[13] M. Randić, "On characterization of molecular branching," Journal of the American Chemical Society, vol. 97, no. 23, pp. 6609-6615, 1975.

[14] B. Zhou and N. Trinajstic, "On a novel connectivity index," Journal of Mathematical Chemistry, vol. 46, no. 4, pp. 1252-1270, 2009.

[15] B. Zhou and N. Trinajstić, "On general sum-connectivity index," Journal of Mathematical Chemistry, vol. 47, no. 1, pp. 210-218, 2010.

[16] D. Vukičević and B. Furtula, "Topological index based on the ratios of geometrical and arithmetical means of end-vertex degrees of edges," Journal of Mathematical Chemistry, vol. 46, no. 4, pp. 1369-1376, 2009.

[17] S. Chen and W. Liu, "The geometric-arithmetic index of nanotubes," Journal of Computational and Theoretical Nanoscience, vol. 7, no. 10, pp. 1993-1995, 2010.

[18] K. C. Das and N. Trinajstić, "Comparision between first geometric-arithmetic index and atom-bond connectivity index," Chemical Physics Letters, vol. 497, no. 1-3, pp. 149-151, 2010.
[19] L. Xiao, S. Chen, Z. Guo, and Q. Chen, "The geometricarithmetic index of benzenoid systems and phenylenes," International Journal of Contemporary Mathematical Sciences, vol. 5, no. 45-48, pp. 2225-2230, 2010.

[20] A. Graovac, M. Ghorbani, and M. A. Hosseinzadeh, "Computing fifth Geometric-Arithmetic index for nanostar dendrimers," Journal of Mahematical Nanoscience, vol. 1, no. 1, pp. 33-42, 2011. 

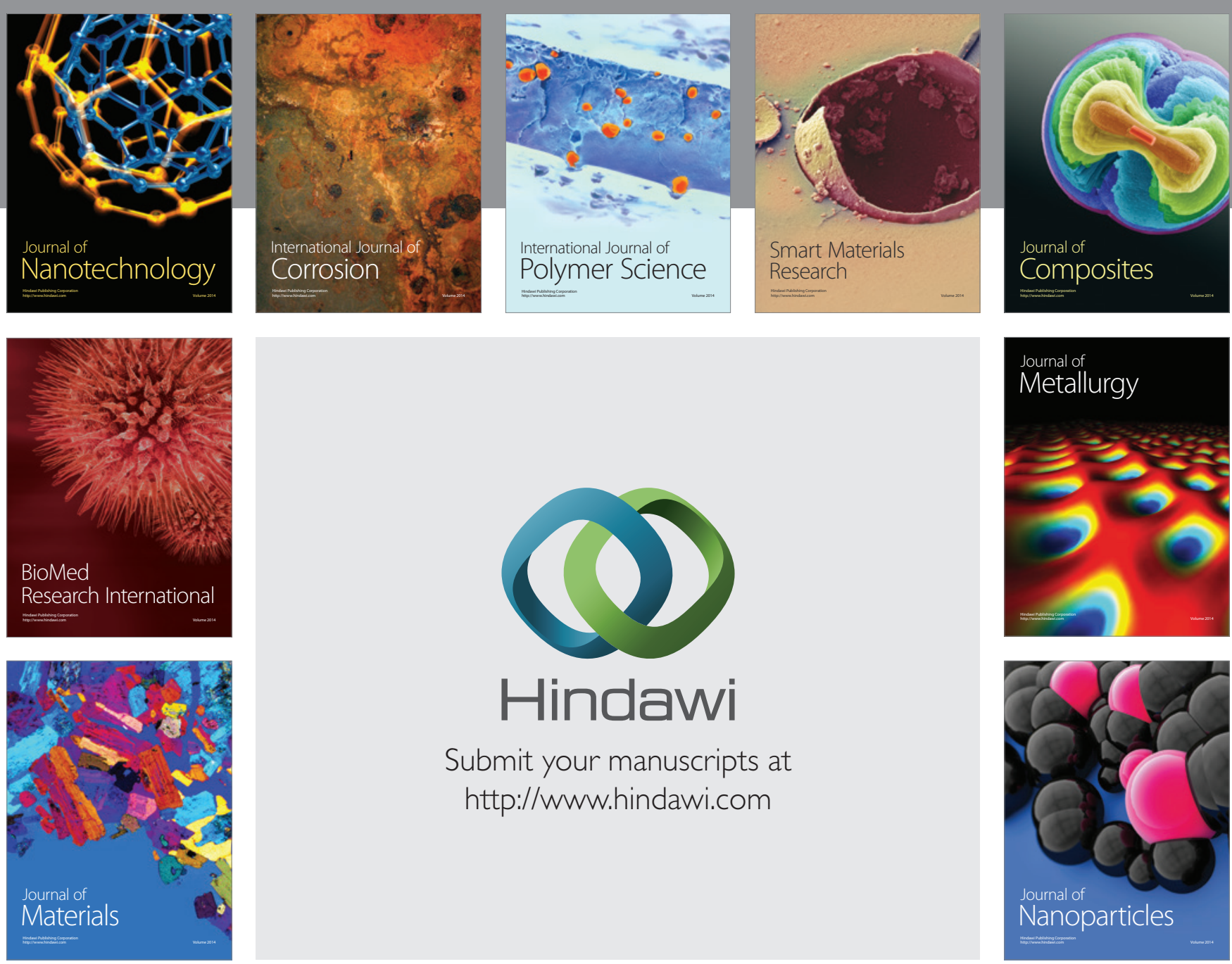

Submit your manuscripts at http://www.hindawi.com
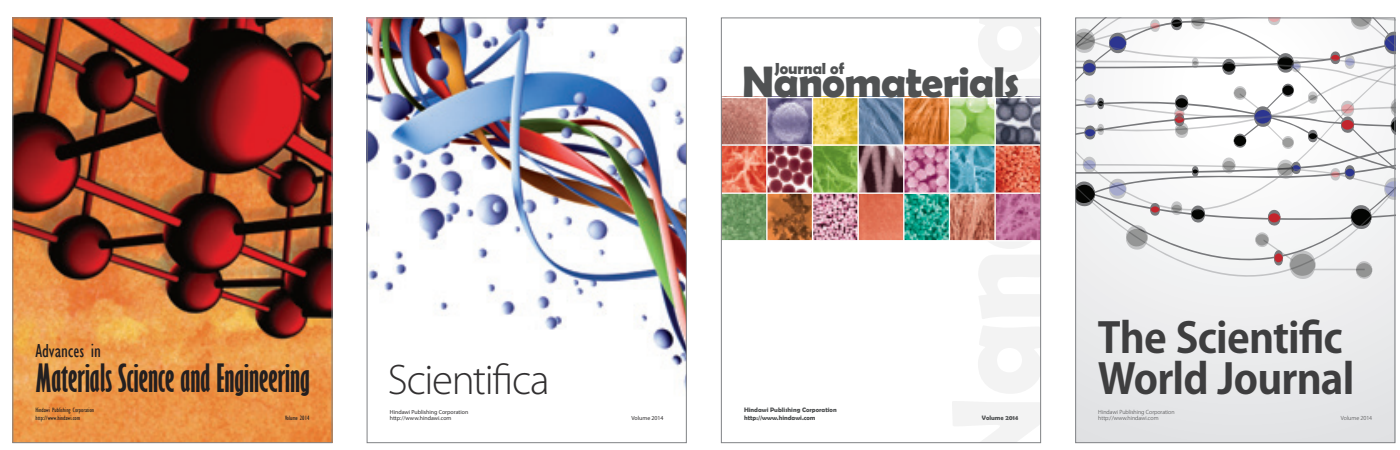

\section{The Scientific World Journal}
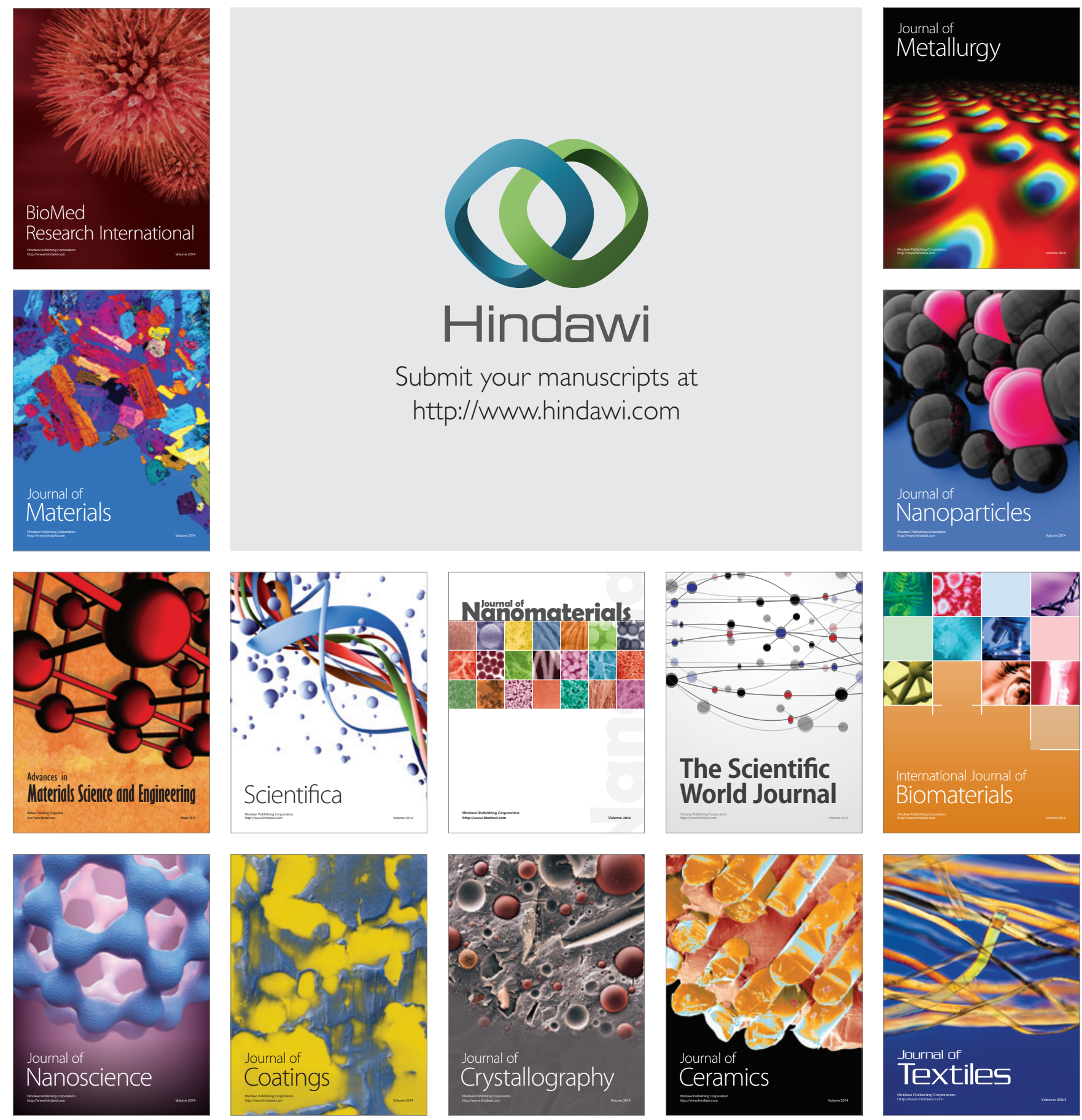